\title{
Targeted Pharmacological Heme-0xygenase-1 Induction as a Therapy for Diabetes
}

\author{
Luc Rochette*, Alexandre Meloux, Eve Rigal and Catherine Vergely \\ Physiopathologie et Epidémiologie Cérébro-Cardiovasculaires (PEC2), Faculté des Sciences de Santé, Université de Bourgogne, France
}

Submission: February 23, 2018; Published: April 11, 2018

*Corresponding author: Luc Rochette, Equipe d’Accueil (EA 7460): Physiopathologie et Epidémiologie Cérébro-Cardiovasculaires (PEC2), Faculté des Sciences de Santé, Université de Bourgogne-Franche Comté, 7 Bd Jeanne d'Arc, 21000 Dijon, France, Tel: +33-380393291; Fax: +33-380393293; Email: luc.rochette@u-bourgogne.fr

Abstract

Diabetes has emerged as a major threat to health worldwide. The exact mechanisms underlying the disease are unknown; however, there is growing evidence that excess generation of reactive oxygen species (ROS), causes oxidative stress in various organs. In diabetic patients, oxidative stress is closely associated with chronic inflammation and plays a key role in the pathogenesis of micro-and macrovascular diabetic complications. Redox reactions associated with carbon monoxide (CO) metabolism play key roles in intra- and inter-cellular signaling. Cells produce significant amounts of $\mathrm{CO}$ as a product of cellular metabolism, largely from heme degradation catalyzed by microsomal heme oxygenases (HOs) generating CO, biliverdin, bilirubin and iron. This review focuses on the importance of both HO-1/CO system in the pathophysiology and therapy of inflammation associated with diabetes. Research on these pathways will open new perspectives for the rational design of drugs against diabetic diseases.

Keywords: Diabetes; Oxidative stress; Reactive oxygen species; Heme-oxygenase-1; Carbon monoxide

\section{Introduction}

Diabetes is a chronic disease characterized by elevated blood sugar levels resulting from either a lack of insulin production or resistance to insulin. About 230 million people worldwide had diabetes in 2010. The global figure of people with diabetes is projected to increase to 333 million in 2025, and 430 million in 2030 [1]. The majority of diabetes patients are not insulindependent and able, at least initially, to produce the hormone. This type of diabetes mellitus (DM) is termed type 2 diabetes. Insulin resistance is a fundamental aspect of the etiology of type 2 diabetes. Subjects with diabetes have an increased risk of ischemic heart disease, atherosclerosis and nephropathy [2,3]. Obesity, which is a major public health concern worldwide, increases the risk of type- 2 diabetes [3]. Type 2 diabetes is caused by a combination of insulin resistance coupled with insufficient production of insulin to overcome the insulin resistance [4]. Oxidative stress plays a key role in the pathogenesis of micro-and macrovascular diabetic complications. There is now convincing evidence that redox reactions associated with $\mathrm{CO}$ metabolism play key roles in adaptive processes of tissues towards oxidative stress. Cells and tissues produce significant amounts of CO from heme degradation catalyzed by microsomal heme oxygenases (HO).

\section{Heme Proteins as Signaling Molecules}

Heme proteins play a major role in various biological functions and most of the reactions involving heme are redox reactions of heme iron. Heme is released from hemoproteins during red blood cell (RBC) destruction and is metabolized by heme oxygenases (HO). Three isoforms of HO have been characterized: an inducible form (HO-1), which is up-regulated, especially in the spleen and liver, in response to various types of stress, and two constitutive forms (HO-2 and HO-3). HO-1 generates signaling molecules through the catalysis of heme-carbon monoxide (CO), biliverdin, bilirubin and iron-each of which acts via distinct molecular targets to influence cell function, both proximally and distally. An excess of heme is deleterious to cells. The damage caused is due to its iron-induced prooxidant effects on all of the compounds of cells; these toxic effects are caused by iron catalyzing the Fenton reaction. Biliverdin reductase (BVR) has two isoforms: BVR-A and BVR-B. Through the activity of BVR, BV is immediately reduced to bilirubin. Extensive research has shown that the HO and BVR systems are closely involved in the molecular regulation of various pathophysiological processes, in particular in cellular adaptation to oxidative stress, and the anti-inflammatory response. In some circumstances, normal homeostatic regulatory mechanisms may be overwhelmed by the production of reactive oxygen species (ROS) and reactive nitrogen species (RNS) such as nitric oxide $(\bullet N O)$ with input from co-factors. The major antioxidant enzymes possess transition metals, selenium, manganese, riboflavin or ubiquinone at the catalytic site and the availability of cofactors can determine the activity of some enzymes [5]. Susceptibility to 
oxidative processes increases with age and with disease as a result of the deterioration of normal physiological control [6]. There is now convincing evidence that redox reactions associated with NO and CO metabolism play key roles in intra-and inter-cellular signaling, and in adaptive processes of tissues towards stress [7]. It is now well recognized that HO-mediated heme degradation has multiple roles, including antioxidant and iron reutilization functions. HO generates the effector molecules biliverdin/ bilirubin, carbon monoxide, and free iron/ferritin.

\section{Oxidative and Nitroxidative Stress in Diabetes}

Given the multiplicity of their functions, mitochondria are a logical target for the study of metabolic diseases. Skeletal muscle is the major site of insulin-stimulated glucose use in the body, and the dysregulation of mitochondria is closely associated with insulin resistance in skeletal muscle and thus with the pathogenesis of type 2 diabetes. Inside mitochondria, electrons from reduced substrates move from complexes I and II of the electron transport chain through complexes III and IV to oxygen, forming water and causing protons to be pumped across the mitochondrial inner membrane. The electron transport system is organized so that the level of ATP can be precisely regulated [8].

The increased superoxide anion production is associated with the activation of major pathways involved in the pathogenesis of diabetic complications: 1) polyol pathway flux, 2) increased formation of AGEs, 3) activation of protein kinase C isoforms, and 4) over-activity of the hexosamine pathway. The accelerated flux of sorbitol through the polyol pathway has been implicated in the pathogenesis of secondary diabetic complications: cataractogenesis, retinopathy, neuropathy, nephropathy and cardiovascular diseases. In addition, in diabetic rats, levels of free carnitine and myo-inositol in the caudal nerves are decreased while polyol accumulates. These actions are accompanied by the inactivation of enzymes such as eNOS [9]. Insulin exerts redoxregulating actions in various target organs, implying that the hormone has an antioxidative role [10]. The generation of ROS by mitochondrial oxidative phosphorylation is attenuated by insulin through the regulation of uncoupling protein (UCP) expression. In addition, the expression of NADPH oxidases (NOX) is inhibited by insulin [11]. In cultured adipocytes, excess glucose and palmitate generate ROS via NOX4 rather than by mitochondrial oxidation. NOX4 is regulated by both NADPH generated in the pentose phosphate pathway and translocation of NOX4 into lipid rafts, leading to the expression of monocyte chemotactic factors [12].

\section{Heme Oxygenases and Endogenous Production of Carbon Monoxide}

Biological systems rely on heme proteins to carry out a number of basic functions: such as oxygen sensing, electron transport, signal transduction, and antioxidant defense enzymes. Most of these reactions are carried out by redox reactions involving heme iron [13]. Heme biosynthesis includes several steps. The first and the last three steps occur in mitochondria; while the others take place in the cytoplasm [14]. Heme is released from hemoproteins during red blood cell (RBC) destruction and metabolized by HO. The majority of heme degradation products are derived from the catabolism of hemoglobin released from senescent RBCs, phagocytosed, and destroyed by the reticuloendothelial (RE) system, primarily in the spleen and liver. The oxidation of heme by the HOs requires the concerted activity of nicotinamide adenine dinucleotide phosphate (NADPH)-cytP450 reductase to provide reducing equivalents to support the reduced state of iron $\left(\mathrm{Fe}^{2+}\right)$ and to activate molecular oxygen [15]. Humans possess control mechanisms to maintain iron homeostasis by coordinately regulating iron absorption, iron recycling, and mobilization of stored iron [16]. In humans, endogenous $\mathrm{CO}$ arises principally from the action of $\mathrm{HO}$, which catalyzes the rate-limiting step in heme degradation. The $\mathrm{HO}$ reaction generates one molecule of $\mathrm{CO}$ per molecule of oxidized heme. HOs play an important physiological role in hemoglobin turnover in reticulo-endothelial tissues such as the spleen, kidney and liver where senescent erythrocytes are destroyed [17].

\section{Functions of Heme Oxygenases}

Three isoforms of $\mathrm{HO}$ have been characterized: an inducible form (HO-1), which is up-regulated, in response to various types of stress, and two constitutive forms (HO-2 and HO-3).

\section{Heme oxygenase-1: HO-1}

The inducible form of HO, HO-1, occurs at a high level of expression in the spleen and other tissues that degrade senescent red blood cells, including specialized reticulo-endothelial cells of the liver and bone marrow. HO-1 is also present in myeloid cells. These cells comprise monocytes, macrophages and dendritic cells, which play crucial regulatory roles in the innate and adaptive immune system. As the liver plays a crucial role in the body's iron homeostasis (e.g. via secretion of the iron regulatory hormone: hepcidin) and in systemic inflammation, hepatic HO-1 may be important for the regulation of both systems. In an organ such as the liver, the induction of HO-1 expression is an important aspect of the anti-inflammatory, anti-apoptotic response to cellular stress. The gene coding for HO- 1 is highly regulated $[18,19]$. HO-1 is emerging as a great potential therapeutic target for treating cardiovascular diseases. In the vascular system, HO-1 and heme degradation products perform essential physiological functions [20]. There appears to be a relationship between HO-1 expression and the signaling pathways that modulate inflammatory response [21]. Nitrated fatty acids (NO2-FA) resulting from interactions between NO and eicosanoid have distinct anti-inflammatory signaling properties. Nitrated linoleic acid potently induces HO-1 expression by an NO- and PPAR $\gamma$-independent mechanism in human aortic endothelial cells [22]. These pathways may converge via the generation of nitrated unsaturated lipids that influence PMN activity and the evolution of inflammation [23].

\section{Heme oxygenase-2: (HO-2)}

HO-2 is constitutively expressed in selected tissues (brain, liver, and testes) and is involved in signaling and regulatory processes. HO-2 has three cysteine residues that are thought 
to modulate the affinity for heme, whereas HO-1 has none [24]. Within the normal liver, HO-2 is constitutively expressed within hepatocytes, Kupffer cells, endothelial cells and Ito cells. In the central nervous system, it has been demonstrated that HO-2 can function as an $\mathrm{O}_{2}$ sensor in the brain, and the $\mathrm{O}_{2}-\mathrm{CO}-\mathrm{H}_{2} \mathrm{~S}$ cascade rapidly mediates hypoxia-induced cerebral vasodilation [25].

\section{Heme oxygenase-3: (HO-3)}

The existence of a third $\mathrm{HO}$ isoform, HO-3, was reported in the rat. HO-3 was shown to be the product of a single transcript of $2.4 \mathrm{~kb}$ encoding a protein of $33 \mathrm{kDa}$. The HO-3 transcript was found in a series of organs including spleen, liver, kidney and brain [26]. The function of HO-3 remains unclear, but it has been cloned from rat brain, suggesting a neural function. This enzyme is structurally similar to HO-2, but is less efficient at degrading heme.

\section{Incidence of Endogenous HO-1 Activation}

The incidence of endogenous HO-1 activation has been studied in experimental and clinical procedures. HO-1 activity provides a possible antioxidative function by accelerating the removal of heme to limit oxidative stress sustained through heme-iron dependent mechanisms. The effects of $\mathrm{CO}$ and bilirubin indirectly reproduce the incidence of $\mathrm{HO}$ activation. Great attention has been paid to the protective role of $\mathrm{CO}$ and carbon monoxide-releasing molecules (CORMs) in vascular diseases. Indeed, $\mathrm{CO}$ and CORMs exert anti-inflammatory and anti-oxidant actions on different organs $[27,28]$. Bilirubin appears to be a more potent antioxidant than biliverdin. Nonetheless, there is evidence that the direct and indirect antioxidant effects of both bile pigments contribute to the beneficial profile of the HO-1 pathway. Individuals with Gilbert's syndrome have polymorphism in the bilirubin UDP-glucuronosyltransferase (UGT1A1) promoter and are protected against a number of factors associated with cardiovascular complications. This polymorphism results in

Table 1: Chemical structures of heme oxygenase inducers. slower glucuronidation and therefore diminished excretion of bilirubin, leading to elevated bilirubin levels in the plasma.

Recent studies have revealed that HO-1 mediates the adiponectin-induced anti-inflammatory response; adiponectin inducing an HO-induction [29]. Adiponectin, an adipokine predominantly secreted from adipocytes, plays a modulatory role in various pathophysiological conditions. Apart from its well characterized role in glucose and fatty acid metabolism, adiponectin has received special attention in recent years due to its protective role in inflammation. Moreover, chronic HO-1 induction also modifies the phenotype of adipocytes in obesity from large, cytokine-producing adipocytes to smaller, adiponectin-producing adipocytes [30]. Emerging evidence indicates that links exist between $\mathrm{HO}$ activity and the changes in energy metabolism that occur during the development of certain diseases. Experimental evidence suggests that excessive amounts of free fatty acids and high glucose produce hypertrophied adipocytes resulting in detrimental perturbations in both mitochondrial and endoplasmatic reticulum function. These effects are associated with the increased generation of ROS, activation of the inflammatory cascade and insulin resistance. The levels of HO-1 expression, HO activity and its products, CO and bilirubin, are decreased in humans and in animal models of type-2 diabetes [31]. In conclusion, the induction of HO-1 appears to modulate metabolic syndrome, obesity, and insulin resistance, and recent data provide evidence for the involvement of the HOadiponectin-EET axis in adipogenesis and adipocyte signaling both in vitro and in vivo [32].

\section{Heme-0xygenases Inducers (Table 1)}

A lot of natural agents have been recognized for their capacity to induce HO-1 in different tissues. Most of these compounds are characterized by a phenolic structure, similar to that of alphatocopherol, and present antioxidant properties.

Curcumin and analogues




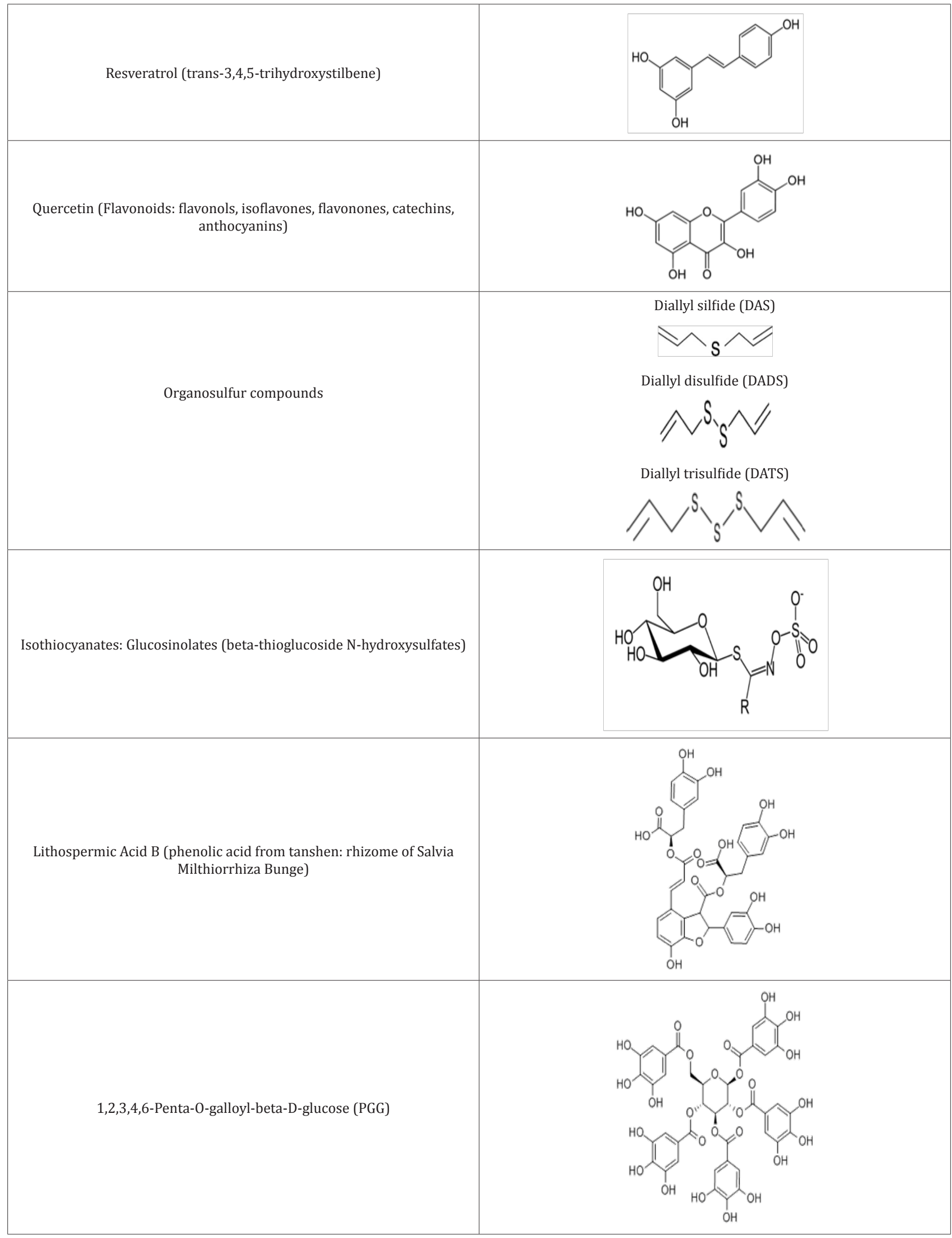




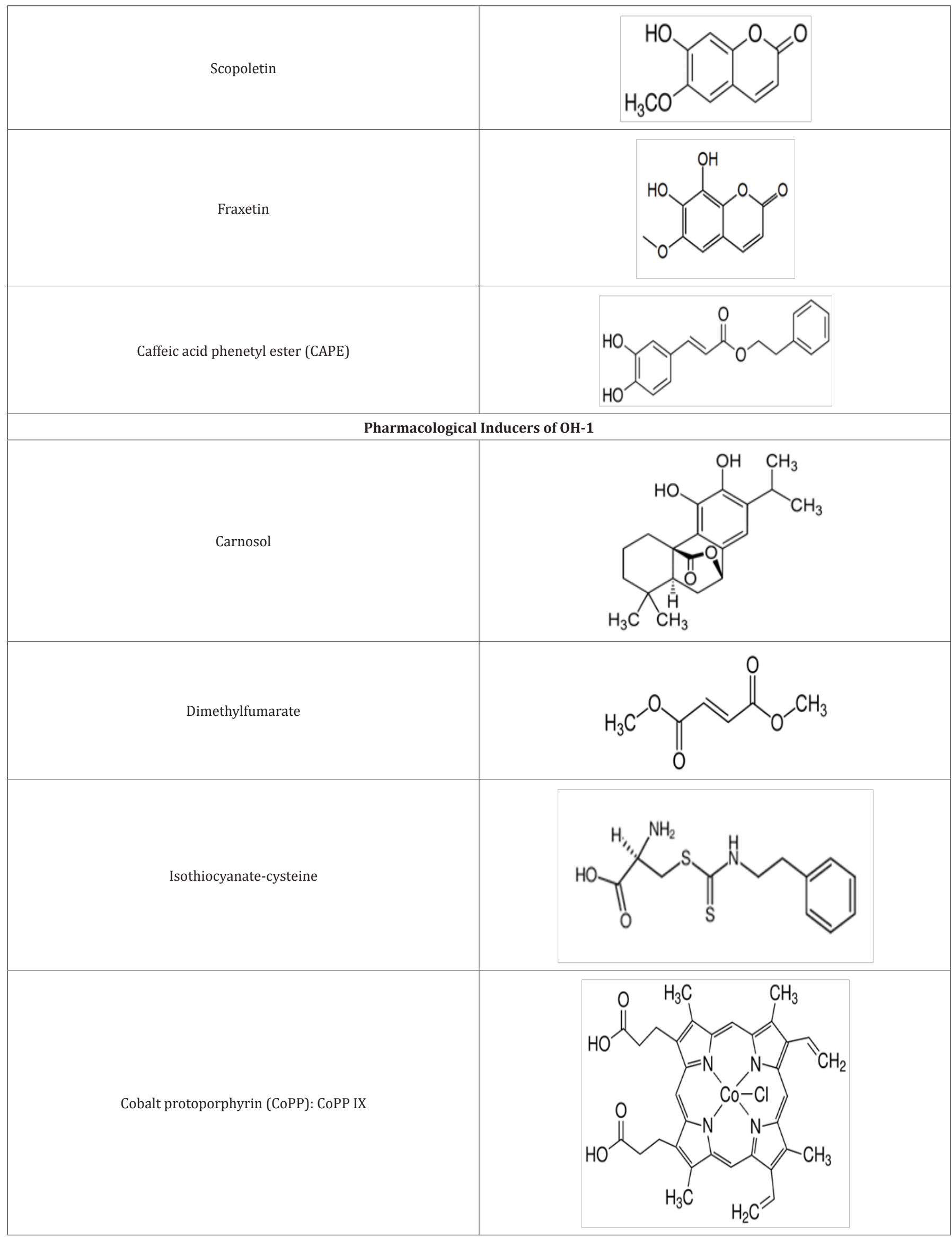




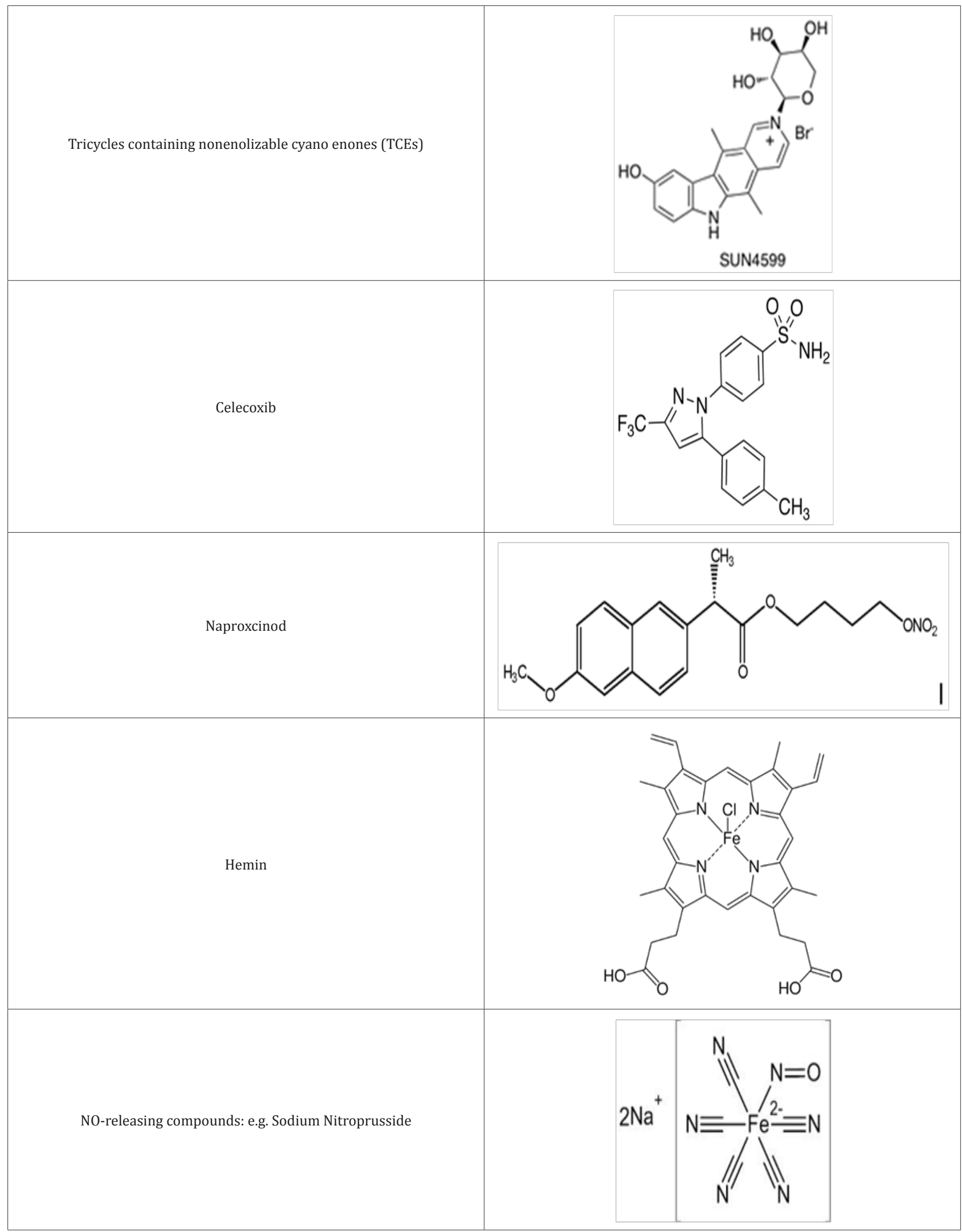




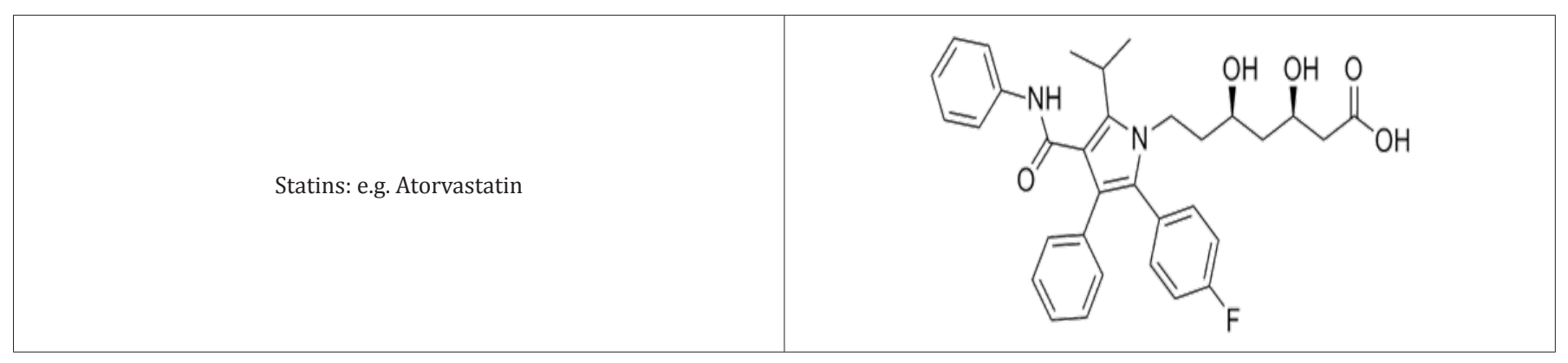

\section{Natural heme-oxygenase-1 inducers}

A number of natural antioxidant compounds contained in foods and plants have been demonstrated to be effective noncytotoxic inducers of the response protein HO-1 in various cellular models. Most of these compounds that induce HO-1 are characterized by phenolic structures and it is speculated that Nrf2 is involved in this induction of HO-1 [33]. The effects of various concentrations of a natural polyphenolic stilbene, resveratrol, on HO activity and HO-1 protein expression in different experimental conditions have been tested. Resveratrol is a nonflavonoid compound produced naturally by plants including grapes, peanuts, cranberries and blueberries. Resveratrol is the major polyphenol in red wine and has been shown to prevent or slow the progression of a wide variety of diseases [34]. The most extensively investigated HO-1 inducer is another natural compound, curcumin (diferuloylmethane). The effects of curcumin are associated with cellular protection against ROS. The level of HO-1 expression was found to highest with curcumin, followed by demethoxycurcumin and bis-demethoxycurcumin. It has been suggested that the presence of methoxyl groups in the ortho-position on the aromatic ring are essential to enhance HO-1 expression [35].

\section{Pharmacological interest approach of HO-1 inducers}

Manipulation of the Nrf2/HO-1 pathway has been shown experimentally to protect against a variety of conditions characterized by oxidative damage and inflammation. Pharmacologically-active compounds have been used to target Nrf2/HO-1. Potent activators of the Nrf2/HO-1 pathway (i.e. carnosol, cobalt protoporphyrin, dimelthyl fumarate) have been shown to modulate inflammation in mouse microglial cells [36]. Metalloporphyrins, particularly cobalt protoporphyrin (CoPP) can increase the expression of HO-1. CoPP affects the expression of antioxidant genes and recent data indicate that CoPP reduces mitochondrial production mediated by Foxo1 [37]. A large number of clinical and experimental pharmacological compounds have been shown to induce HO-1, via NO metabolism. The different statins with established antiatherogenic or cardioprotective activities are able to induce HO-1 [38]. NOreleasing compounds, such as sodium nitroprusside, S-nitroso- $\mathrm{N}$ acetylpenicillamine, and 3-morpholinosydnonimine, induce HO-1 in endothelial cells [39]. Studies suggest that aspirin may exert part of its antiinflammatory effect via the NO-mediated induction of HO-1 [40]. The increase in HO-1 expression in response to other compounds is the result of a complex regulatory network involving many signaling pathways and transcription factors. Pharmacological doses of insulin have been reported to induce HO-1 in renal cells via the phosphatidylinositol 3-kinase/Akt pathway and Nrf2 and this may represent a mechanism by which insulin protects the kidney in addition to its effect on circulating glucose concentration [41,42].

\section{Conclusion}

Extensive research has shown that the HO system is closely involved in the regulation of various pathophysiological processes, in particular in cellular adaptation to oxidative stress, and the anti-inflammatory response. It is now well recognized that HO-mediated heme degradation has multiple roles, including antioxidant and iron reutilization functions. The multiple cytoprotective mechanisms of HO-1 make it a promising therapeutic target. Regulation of HO-1 activity may be a therapeutic strategy for a number of inflammatory conditions and it may be important to explore the overall protective roles of the HO-1/CO system in the pathogenesis of human cardiovascular and vascular diseases.

\section{Acknowledgment}

This work was supported by grants from French Ministry of Research, Inserm (Institut national de la santé et de la recherche médicale) and, from the Regional Council of Burgundy Franche Comte (Conseil Régional de Bourgogne et de Franche Comté), FEDER and Association de Cardiologie de Bourgogne.

\section{References}

1. Wild S, Roglic G, Green A, Sicree R, King H (2004) Global prevalence of diabetes: estimates for the year 2000 and projections for 2030 . Diabetes care 27(5): 1047-1053.

2. Mazzone T, Chait A, Plutzky J (2008) Cardiovascular disease risk in type 2 diabetes mellitus: insights from mechanistic studies. Lancet 371(9626): 1800-1809.

3. Zeller M, Steg PG, Ravisy J, Laurent Y, Janin ML, et al. (2005) Prevalence and impact of metabolic syndrome on hospital outcomes in acute myocardial infarction. Arch Intern Med 165(10): 1192-1198.

4. Graves DT, Kayal RA (2008) Diabetic complications and dysregulated innate immunity. Front Biosci 13: 1227-1239.

5. Orsucci D, Filosto M, Siciliano G, Mancuso M (2009) Electron transfer mediators and other metabolites and cofactors in the treatment of mitochondrial dysfunction. Nutrition reviews 67: 427-438. 
6. Balaban RS, Nemoto S, Finkel T (2005) Mitochondria, oxidants, and aging. Cell 120(4): 483-495.

7. Lamon BD, Zhang FF, Puri N, Brodsky SV, Goligorsky MS, et al. (2009) Dual pathways of carbon monoxide-mediated vasoregulation: modulation by redox mechanisms. Circ Res 105(8): 775-783.

8. Treberg JR, Brand MD (2011) A model of the proton translocation mechanism of complex I. J Biol Chem 286(20): 17579-17584.

9. Giacco F, Brownlee M (2010) Oxidative stress and diabetic complications. Circ Res 107(9): 1058-1070.

10. Wang X, Tao L, Hai CX (2012) Redox-regulating role of insulin: the essence of insulin effect. Mol Cell Endocrinol 349(2): 111-127.

11. Newsholme P, Haber EP, Hirabara SM, Rebelato EL, Procopio J, et al. (2007) Diabetes associated cell stress and dysfunction: role of mitochondrial and non-mitochondrial ROS production and activity. J Physiol 583(1): 9-24.

12. Han CY, Umemoto T, Omer M, Hartigh DLJ, Chiba T, et al. (2012) NADPH oxidase-derived reactive oxygen species increases expression of monocyte chemotactic factor genes in cultured adipocytes. J Biol Chem 287(13): 10379-10393.

13. Michaca LG, Farrugia G, Croatt AJ, Alam J, Nath KA (2004) Heme: a determinant of life and death in renal tubular epithelial cells, American journal of physiology. Am J Physiol Renal Physiol 286(2): F370-F377.

14. Woodard SI, Dailey HA (2000) Multiple regulatory steps in erythroid heme biosynthesis. Arch Biochem Biophys 384(2): 375-378.

15. Krishnamurthy P, Xie T, Schuetz JD (2007) The role of transporters in cellular heme and porphyrin homeostasis. Pharmacol Ther 114(3): 345-358.

16. Zhang AS, Enns CA (2009) Molecular mechanisms of normal iron homeostasis. Hematology Am Soc Hematol Educ Program 2009: $207-$ 214.

17. Ryter SW, Otterbein LE (2004) Carbon monoxide in biology and medicine, Bioessays 26(3): 270-280.

18. Kim HP, Ryter SW, Choi AM (2006) CO as a cellular signaling molecule. Annu Rev Pharmacol Toxicol 46: 411-449.

19. Ryter SW, Alam J, Choi AM (2006) Heme oxygenase-1/carbon monoxide: from basic science to therapeutic applications. Physiol Rev 86(2): 583-650.

20. Wu ML, Ho YC, Yet SF (2011) A central role of heme oxygenase-1 in cardiovascular protection. Antioxid Redox Signal 15(7): 1835-1846.

21. Baker PR, Schopfer FJ, O’Donnell VB, Freeman BA (2009) Convergence of nitric oxide and lipid signaling: anti-inflammatory nitro-fatty acids. Free radical biology \& medicine 46(8): 989-1003.

22. Wright MM, Schopfer FJ, Baker PR, Vidyasagar V, Powell P, et al. (2006) Fatty acid transduction of nitric oxide signaling: nitrolinoleic acid potently activates endothelial heme oxygenase 1 expression. Proc Natl Acad Sci USA 103(11): 4299-4304.

23. Wanikiat P, Woodward DF, Armstrong RA (1997) Investigation of the role of nitric oxide and cyclic GMP in both the activation and inhibition of human neutrophils. Br J Pharmacol 122(6): 1135-1145.

24. Varfaj F, Lampe JN, Montellano PR (2012) Role of cysteine residues in heme binding to human heme oxygenase-2 elucidated by twodimensional NMR spectroscopy. J Biol Chem 287(42): 35181-35191.

25. Morikawa T, Kajimura M, Nakamura T, Hishiki T, Nakanishi T, et al. (2012) Hypoxic regulation of the cerebral microcirculation is mediated by a carbon monoxide-sensitive hydrogen sulfide pathway. Proc Natl Acad Sci USA 109(4): 1293-1298.
26. Hayashi S, Omata Y, Sakamoto H, Higashimoto Y, Hara T, et al. (2004) Characterization of rat heme oxygenase-3 gene. Implication of processed pseudogenes derived from heme oxygenase- 2 gene. Gene 336(2): 241-250.

27. Chung HT, Pae HO, Cha YN (2008) Role of heme oxygenase- 1 in vascular disease. Curr Pharm Des 14(5): 422-428.

28. Przeczek AG, Dulak J, Jozkowicz A (2012) Haem oxygenase-1: noncanonical roles in physiology and pathology. Clin Sci (Lond) 122(3): 93-103.

29. Rochette L, Cottin Y, Zeller M, Vergely C (2013) Carbon monoxide: mechanisms of action and potential clinical implications. Pharmacol Ther 137(2): 133-152.

30. Lin H, Yu CH, Jen CY, Cheng CF, Chou Y, et al. (2010) Adiponectinmediated heme oxygenase-1 induction protects against iron-induced liver injury via a PPA Ralpha dependent mechanism. Am J Pathol 177(4): 1697-1709.

31. Hosick PA, Stec DE (2012) Heme oxygenase, a novel target for the treatment of hypertension and obesity? American journal of physiology. Regulatory. Am J Physiol Regul Integr Comp Physiol 302: R207-R214.

32. Kruger AL, Peterson SJ, Schwartzman ML, Fusco H, McClung JA, et al. (2006) Up-regulation of heme oxygenase provides vascular protection in an animal model of diabetes through its antioxidant and antiapoptotic effects. J Pharmacol Exp Ther 319(3): 1144-1152.

33. Burgess A, Vanella L, Bellner L, Schwartzman ML, Abraham NG (2012) Epoxyeicosatrienoic acids and heme oxygenase-1 interaction attenuates diabetes and metabolic syndrome complications. Prostaglandins Other Lipid Mediat 97(1-2): 1-16.

34. Li C, Hossieny P, Wu BJ, Qawasmeh A, Beck K, et al. (2007) Pharmacologic induction of heme oxygenase-1. Antioxid Redox Signal 9(12): 2227-2239.

35. Baur JA, Sinclair DA (2006) Therapeutic potential of resveratrol: the in vivo evidence. Nat Rev Drug Discov 5(6): 493-506.

36. Son Y, Lee JH, Chung HT, Pae HO (2013) Therapeutic roles of heme oxygenase-1 in metabolic diseases: curcumin and resveratrol analogues as possible inducers of heme oxygenase-1. Oxid Med Cell Longev 2013: 639541.

37. Foresti R, Bains SK, Pitchumony TS, Bras LE, Drago F, et al. (2013) Small molecule activators of the Nrf2-HO-1 antioxidant axis modulate heme metabolism and inflammation in BV2 microglia cells. Pharmacol Res 76: 132-148.

38. Liu X, Cui Y, Li M, Xu H, Zuo J, et al. (2013) Cobalt protoporphyrin induces HO-1 expression mediated partially by FOXO1 and reduces mitochondria-derived reactive oxygen species production. PloS One 8(11): e80521.

39. Grosser N, Hemmerle A, Berndt G, Erdmann K, Hinkelmann U, et al. (2004) The antioxidant defense protein heme oxygenase 1 is a novel target for statins in endothelial cells. Free Radic Biol Med 37(12): 2064-2071.

40. Motterlini R, Foresti R, Intaglietta M, Winslow RM (1996) NO-mediated activation of heme oxygenase: endogenous cytoprotection against oxidative stress to endothelium. Am J Physiol 270(1 Pt 2): H107-H114.

41. Nascimento SV, Arruda MA, Fidalgo BC, Villela CG, Fierro IM (2005) Novel lipid mediator aspirin-triggered lipoxin A4 induces heme oxygenase-1 in endothelial cells. Am J Physiol Cell Physiol 289(3): C557-C563.

42. Harrison EM, McNally SJ, Devey L, Garden OJ, Ross JA, et al. (2006) Insulin induces heme oxygenase- 1 through the phosphatidylinositol 3-kinase/Akt pathway and the Nrf2 transcription factor in renal cells. FEBS J 273(11): 2345-2356. 

Co This work is licensed under Creative
Commons Attribution 4.0 License
DOI: 10.19080/JOCCT.2018.10.555779
Your next submission with Juniper Publishers will reach you the below assets

- Quality Editorial service

- Swift Peer Review

- Reprints availability

- E-prints Service

- Manuscript Podcast for convenient understanding

- Global attainment for your research

- Manuscript accessibility in different formats

( Pdf, E-pub, Full Text, Audio)

- Unceasing customer service

Track the below URL for one-step submission https://juniperpublishers.com/online-submission.php 